\title{
A Sea Clutter Suppression Algorithm by Statistics Estimation and Subspace Analysis
}

\author{
Wenhu Xue, Ling Mao and Tao Liu \\ Electronics Engineering College, Naval University of Engineering, Wuhan, China \\ wenhux@163.com
}

Keywords: subspace; sea clutter; Expectation Maximization algorithm

\begin{abstract}
To solve the problem of target detection in heavy sea clutter, we make simulation study on a subspace-based clutter suppression method to improve signal to clutter ratio in the predicted target location, and thus to improve the detection performance. With the compound Gaussian model of the sea clutter, we first estimate the statistics of sea clutter by Expectation-Maximization (EM) algorithm, then exploit a subspace-based approach to further mitigate sea clutter. With the algorithm, the computational complexity is effectively reduced. Nonetheless the algorithm exhibits good performance of clutter suppression. Numerical results show that the algorithm is effective in sea clutter suppression.
\end{abstract}

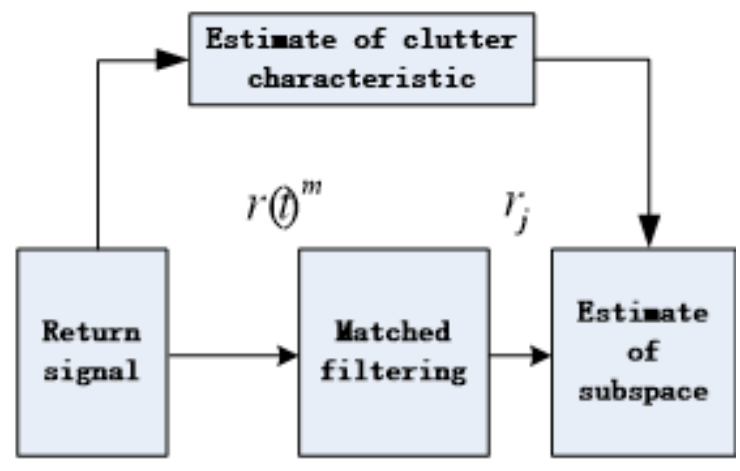

Fig.1 The structure of clutter suppression system.

\section{Introduction}

The detection technology for the sea surface targets with radar is widely used on marine safety, maritime rescue, etc. It is very important in both military and civil areas. The biggest challenge for the detection of small targets on sea surface for surveillance radar is heavy sea clutter. The small targets mainly refer to small boats, sea-skimming missiles and drifting mines drowned in heavy sea clutter. With the normalization of non-war military actions such as naval escort, sovereignty declaration, daily training, etc, the security of our naval ships is often threatened by the small targets. In the environment of low SCR which is caused by low RCS of targets, low grazing angles and high sea states, etc, the function of Radar detection decreases seriously and it can't meet the requirements of application. So how to reduce the influence of sea clutter on targets detection has become one of the hotspots in the field of radar signal processing.

Some progress has been made on using statistical properties of sea clutter to suppress the clutter [1, 2]. For example, methods of application technologies such as time-frequency analysis [3], wavelet transform [4] and neural network [5] have been applied into clutter suppression. However the computation burden of these methods is high in engineering practice and it can't meet the requirement of real time processing for radar. For the sea clutter suppression algorithm which can be used in practical equipments, it must not only fit the requirement of instantaneity, but also has enough good property on clutter suppression. Actually these algorithms are few. Therefore, we present a subspace-based sea clutter suppression method in this paper. It maps the echo signal of sea clutter 
into low order subspace to suppress the clutter [6], and makes radar easily find the targets. Thus the detection performance is improved effectively.

In this paper, firstly we provide the model of echo signal and sea clutter, and estimate the statistical property of sea clutter by Expectation Maximization (EM) algorithm. Secondly, with the subspace method we map the echo signal of sea clutter into low order subspace to suppress the clutter. Finally, we check the validity of the algorithm by numerical simulation.

\section{Model of Echo Signal}

The structure of clutter suppression system is shown in Fig. $1 . K$ pulses of the echo signal constitute a pulse train. With the pulse train, we can get the statistical property of sea clutter. Assuming there are a mass of clutter scattering sources in radar operation environment, and they are distributed uniformly in range-Doppler cells. In one dwell time of the beam, the compound reflection coefficient from the $i$ th clutter scattering sources to $K$ transmitting pulses can be expressed as

$$
\mathbf{y}_{i}=\left[y_{i}^{0}, \ldots, y_{i}^{K-1}\right]^{T}
$$

Supposing in each range cell, the amount of clutter scattering sources, time delay and Doppler shift is constant in dwell times. However, its complex reflection coefficient is randomly fluctuating, because any small change in range (about the scale of radar wave length) may lead to a large change in the phase of echo signal [7].

In Fig. 1, the echo signal $r^{m}(t)$ is:

$$
r^{m}(t)=b^{m} s\left(t-\tau_{0}\right) \exp \left(j 2 \pi v_{0} t\right)+\sum_{i} y_{i}^{m} s\left(t-\tau_{i}\right) \exp \left(j 2 \pi v_{i} t\right)+n(t)
$$

where, $b^{m}, \tau_{0}$ and $v_{0}$ are the target's complex reflection coefficient, time delay and Doppler shift to the $m$ th pulse; $\tau_{i}$ and $v_{i}$ are the time delay and Doppler shift of the $i$ th clutter scattering source; $n(t)$ is additive noise. The target is assumed to be a point target [7], and the fluctuation characteristic meets Swerling I model. The complex reflection coefficients $\mathbf{b}=\left[\boldsymbol{b}^{0}, \boldsymbol{b}^{1}, \ldots, \boldsymbol{b}^{K-1}\right]$ obey Gaussian distribution $\mathbf{b} \sim C N\left(0, \sigma^{2} \mathbf{I}_{K}\right)$, where $\mathbf{I}_{K}$ is a $K \times K$ unit matrix. As we mainly consider the low SCR environment, the covariance of target complex reflection coefficient $\sigma^{2}$ is small, and thus sea clutter becomes the dominant component of the echo signal. Therefore we ignore the influence of additive noise since we only consider the transmitting signal with short duration time, and its Doppler resolution is poor. So we can ignore Doppler processing of the echo.

Sampling $r^{m}(t)$ with the rate of $f_{s}$, we get the sequence $r^{m}[n]=r^{m}\left(n / f_{s}\right)$. The echo signal sequence after matched filtering is expressed by $r_{n}^{m}$. The output vector of the $j$ th time delay or range cell is $\mathbf{r}_{j} \in C^{K \times 1}=\left[r_{j}^{0}, r_{j}^{1}, \ldots, r_{j}^{K-1}\right]^{T}$, the expression is

$$
\mathbf{r}_{j}=\mathbf{b} z_{s}\left[j-n_{0}\right]+\sum_{n=-N_{s}-1}^{N_{s}-1}\left(z_{s}[n] \sum_{i \in \prod_{j+n}} \mathbf{y}_{\mathbf{i}}\right)
$$

where $\Pi_{n}$ is the clutter scattering sources set in the first $n$ range cell, $N_{s}$ is the length of transmitting signal, $\quad n_{0}$ is the index of range cell in which the target is located, $z_{s}[n]$ is the self-correlation function of transmitting signal $s[n]$ defined as

$$
Z_{s}[m]=\sum_{n=0}^{N_{s}-1} s[n] s^{*}[n-m], \quad|m|<N_{s}
$$

Independent scattering sources can be considered as an ultimate situation in which the number of the clutter scattering centre on the sea increases progressively, and it can produce a total effect to the echo signal of sea clutter [8]. Therefore, we regard all the independent scattering sources in each 
range cell as a whole scattering source, therefore the complex scattering coefficient of clutter scattering sources in the $n$th range cell is

$$
\mathbf{y}_{n}=\sum_{i \in \Pi_{n}} \mathbf{y}_{\mathbf{i}}
$$

At the moment, the echo signal can be simplified as:

$$
\mathbf{r}_{j}=\mathbf{b} z_{s}\left[j-n_{0}\right]+\sum_{n=-N_{s}-1}^{N_{s}-1}\left(z_{s}[n] \mathbf{y}_{j+n}\right)
$$

We can see from (6) that we must know the statistical characteristic of sea clutter to process the echo signals. Therefore, it's necessary for us to establish a model which can express the statistical characteristic of sea clutter accurately.

\section{Model of Sea Clutter}

For the early low resolution radar, we commonly suppose that the amplitude of clutter obeys independent identically distributed Gaussian distribution. However, when the resolution is high enough, the sea clutter has non-Gaussian characteristics such as a serious 'Smearing Effect' and asymmetry. Thus the Compound-Gaussian Model [9] with smearing has drawn a great deal of attention. It is appropriate to describe the clutter's statistical characteristic of high resolution radar theoretically and empirically.

According to the Compound-Gaussian Model, sea clutter is consisted of two components. One is small scale structure, and its properties are short related time and spatial independence, named with speckle. Speckle is a rapid fluctuant component, corresponding to the clutter's partial scattering, which obeys Gaussian distribution. It is mainly affected by capillary waves which are close to the sea surface. The other is large scale structure, named with texture. It is a slow fluctuant component, and it can modulate speckle. Texture reflects clutter's local average power, and it is affected by sea states, wind, stream and so on. Also it has longer correlated time.

In Compound-Gaussian Model, the complex scattering reflection of the ith clutter scattering source [10] is

$$
\mathbf{y}_{i}=\sqrt{T_{i}} \mathbf{S}_{i}
$$

where speckle $\mathbf{S}_{i} \in C^{K \times 1}$ is a stationary complex Gaussian process with mean value zero and covariance $\boldsymbol{\Sigma} \in C^{K \times K}$. The texture $T_{i} \geq 0$, is a nonnegative random process. Given the texture $T_{i}$, speckle covariance matrix $\boldsymbol{\Sigma}, \mathbf{y}_{\mathbf{n}} \sim C N\left(0, T_{n} \boldsymbol{\Sigma}\right)$ and the complex scattering coefficients of two scattering sources $\mathbf{y}_{i}$ and $\mathbf{y}_{j}$ are mutually independent [7], we have

$$
p\left(\mathbf{y}_{i}, \mathbf{y}_{j} \mid T_{i}, T_{j}, \boldsymbol{\Sigma}\right)=p\left(\mathbf{y}_{i} \mid T_{i}, \boldsymbol{\Sigma}\right) p\left(\mathbf{y}_{j} \mid T_{j}, \boldsymbol{\Sigma}\right)
$$

Compound-Gaussian Model can not only describe the amplitude measurements characteristic of sea clutter, but also simulate the temporal and spatial correlations of sea clutter. Thus it is appropriate to be used in describing the sea clutter of high resolution radar that is working at low incidence angles and high sea states [7].

\section{Statistics Estimation of Clutter}

The existing statistics estimation of clutter are mostly realized by estimating the correlation parameters of clutter models [11]. Here we directly estimate the texture of clutter and the covariance matrix of speckle $\boldsymbol{\Sigma}$ in Compound Gaussian Model. 
Assuming the target is located in the $j$ th range cell, then the clutter's statistics characteristic can be estimated as

$$
\Theta=\left\{T_{j-2\left(N_{s}-1\right)}, \ldots, T_{j}, \ldots, T_{j+2\left(N_{s}-1\right)}, \Sigma\right\}
$$

Although texture is a random process, we have supposed that it is constant in the whole dwell time, and it is a definite but unknown variable in each range cell.

It's a multiple to one mapping between the complex scattering coefficient of clutter and the output vector of matched filter, and it's irreversible. Therefore, given the echo signal, we can only get the maximum likelihood estimate of $\Theta$. The observation data of radar can be expressed as:

$$
\mathbf{r}=\left[\mathbf{r}_{j-(N-1)}, \ldots, \mathbf{r}_{j}, \ldots, \mathbf{r}_{j+(N-1)}\right]^{H}
$$

Then the maximum likelihood estimation of $\Theta$ is

$$
\hat{\Theta}=\arg \max \underset{\Theta}{p}(\mathbf{r} ; \Theta)
$$

where $p(\mathbf{r} ; \Theta)$ is the probability density function of observation vector $\mathbf{r}$ which is depended on clutter's estimator $\Theta$. The maximization operation in (11) is complicated multi-dimensional searching, and it's hard to calculate. Using EM algorithm instead of multi-dimensional searching to estimate $\Theta$ can reduce the computation load. Using an unobserved self-contained data instead of an observed uncompleted data vector $\mathbf{r}$, the unobserved self-contained data is expressed as

$$
\mathbf{y}=\left[\tilde{\mathbf{y}}_{j-2\left(N_{s}-1\right)}, \ldots, \tilde{\mathbf{y}}_{j}, \ldots, \tilde{\mathbf{y}}_{j+2\left(N_{s}-1\right)}\right]^{H}
$$

where $\mathbf{y}_{j}=\mathbf{y}+\mathbf{b} \delta\left[j-n_{0}\right], \quad \delta[\cdot]$ is Kronecker function, $\mathbf{b}$ is the complex reflection coefficient of target, $\mathbf{y}_{j}$ is the clutter's complex reflection coefficient in the $j$ th range cell. Then the maximum likelihood estimation of $\Theta$ is

$$
\hat{\Theta}=\arg \max \underset{\Theta}{p}(\mathbf{y} ; \Theta)
$$

Here we can use EM algorithm in [12] to estimate the time-varying statistical characteristic of sea clutter.

\section{Clutter Suppression Algorithm}

Subspace-based Clutter Suppressions. The subspace method for clutter suppression generally assumes that most power is concentrate on a low order subspace [12]. By mapping the echo signal orthogonally to the low order subspace of clutter, we get the orthogonal signal. In the orthogonal signal, the ratio of clutter decreases and then the target SCR increases obviously.

The covariance of echo signal $\mathbf{r}_{j}$ is

$$
\begin{aligned}
\mathbf{R}_{j}= & E\left\{\mathbf{r}_{j} \mathbf{r}_{j}^{H}\right\} \\
= & E\left\{\mathbf{b} \mathbf{b}^{H}\right\}\left|z_{s}\left[j-n_{0}\right]\right|^{2}+ \\
& \sum_{n=-\left(N_{s}-1\right)}^{N_{s}-1} \sum_{i=-\left(N_{s}-1\right)}^{N_{s}-1} E\left\{\mathbf{y}_{j+n} \mathbf{y}_{j+i}^{H}\right\} z_{s}[n] z_{s}^{*}[i]
\end{aligned}
$$

Because the complex reflection coefficients of target meet $\mathbf{b} \sim C N\left(0, \sigma^{2} \mathbf{I}_{K}\right)$, the model of sea clutter is a Compound-Gaussian Model, and then (14) can be simplified as 


$$
\begin{aligned}
\mathbf{R}_{j} & =\sigma^{2} I_{K}\left|z_{s}\left[j-n_{0}\right]\right|^{2}+\sum_{n=-\left(N_{s}-1\right)}^{N_{s}-1} T_{j+n} \sum\left|z_{s}[n]\right|^{2} \\
& =\sigma^{2} I_{K}\left|z_{s}\left[j-n_{0}\right]\right|^{2}+\sum \beta_{j}
\end{aligned}
$$

where scalar $\beta_{j}=\sum_{n=-\left(N_{s}-1\right)}^{N_{s}-1} T_{j+n}\left|z_{s}[n]\right|^{2}$ is a function which is decided by the transmitting waveform.

From (15) we can see that the feature space of sea clutter is the same as that of speckle. Therefore the feature space of sea clutter can be obtained from estimated eigen-decomposition of $\hat{\boldsymbol{\Sigma}}$.

Let the matrix $Q_{c} \in K \times N_{e}$, its column vector is the eigenvector of $\sum$, and $N_{e}<K$ ( $K$ is the number of eigenvalues for $\boldsymbol{\Sigma}$ ). Then $Q_{c}^{\perp} \in K \times K^{\prime}, K^{\prime}=K-N_{e}$. The column vector is the remaining feature vector of $\boldsymbol{\Sigma}$. Then, by orthogonally mapping the echo signal to the low order subspace of clutter, we can decrease the power of sea clutter and get the echo signal after clutter suppression as

$$
\mathbf{r}_{j}^{\perp}=Q_{c}^{\perp^{H}} \mathbf{r}_{j}
$$

Numerical Simulation. The sea clutter component in the echo signal $\mathbf{r}_{j}$ is contributed by about 4000 clutter scattering sources, and there are averagely 20 clutter scattering sources in each range cell. They are uniformly distributed in Doppler frequency $[-1,1] \mathrm{kHz}$. The transmitting signal $s(t)$ is LFM pulse with fixed parameters whose bandwidth is $50 \mathrm{MHz}$, and the pulse width is $2 \mu$ s. In addition, the transmitted pulse have unit energy, and $K=10$. The pulse repeat interval is $100 \mu \mathrm{s}$, and so the length of each sub-dwell time is $1 \mathrm{~ms}$. In the de-correlated time of the speckle, the varying of sea clutter statistics is not much. The sampling frequency $f_{s}$ is $50 \mathrm{MHz}$, and the number of sampling points is $N_{s}=100$. The complex reflection coefficient of target echo is derived by sampling from a zero-mean complex Gaussian process whose covariance matrix is $\sigma^{2} \mathbf{I}_{k}$.

After getting the statistical characteristic estimate of sea clutter, we use the sub-space method to implement clutter suppression. Fig. 2 shows the echo signal after clutter suppression, where $\left\|\mathbf{r}_{j}\right\|_{2}$ and $\left\|\mathbf{r}_{j}^{\perp}\right\|_{2}$ are the 2-norm or modulus of the original echo signal and the echo after clutter suppression, which are in the range cell where the target is located and its 200 adjacent range cells.
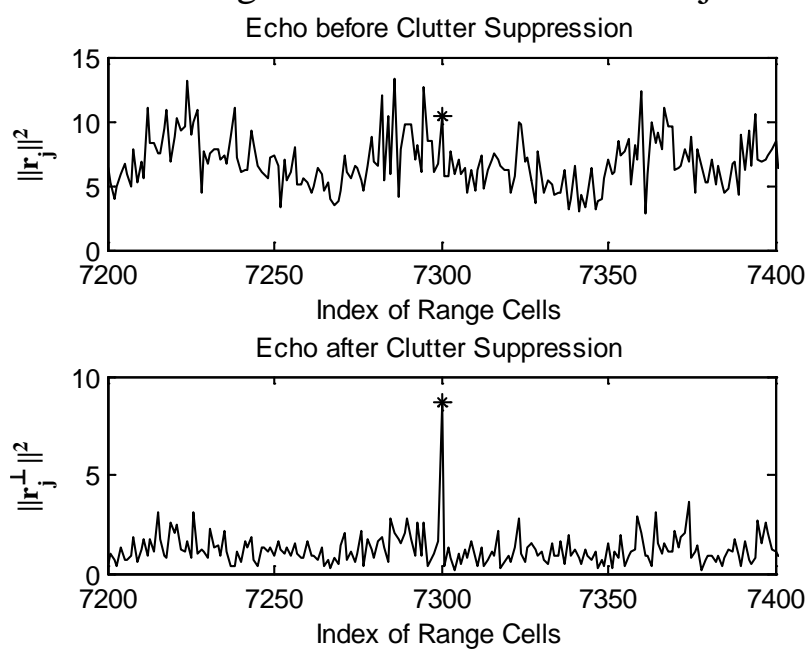

Fig.2 Comparison between echo signals before and after clutter suppression.

The asterisk symbol $(*)$ in Fig. 2 indicates the range cell which the target is located in. In the original echo signal, the target is drowned in sea clutter. If we do not use the clutter suppression 
algorithm, it's hard to find the target. However after clutter suppression, the signal to noise ratio (SNR) is improved obviously. Simple matrix operation is used in the subspace-based method, and thus the computation burden is not hard. Thus it's good to improve the detection performance for the targets drowned in sea clutter. Consequently, through simulation results we can see that the algorithm based on statistics estimation and subspace method is effective in suppression of sea clutter.

\section{Conclusion}

We study a subspace-based clutter suppression method to improve SNR of radar echo wave in this paper. With compound-Gaussian model to describe the clutter statistical characteristics which is in low incident angle and high altitude condition, and using EM algorithm to estimate the clutter statistics which is in the predicted target location and its adjacent cells. Using maximum likelihood estimation to get the expression of low order subspace of clutter, and orthogonally mapping the echo signal to the subspace, we obtain the orthogonal signal with clutter well suppressed. The signal can not only increase the SCR of the range cell where the target is located, but also decrease the computation load by mapping the data to a low order subspace. Simulation results show that the algorithm is effective to be used for sea clutter suppression.

\section{References}

[1] K. Ward, C. Baker, S. Watts. Maritime surveillance radar Part I: Radar scattering from the ocean surface. IEEE Proceedings F: Communications, Radar and Signal Processing, 1990,137: 51-62.

[2] S. Haykin, R. Bakker, B.W. Currie. Uncovering nonlinear dynamics - The case study of sea clutter. Proceedings of the IEEE, 2002,90: 860-881.

[3] T. Thayaparan. Detection of a manoeuvring air target in seaclutter using joint time-frequency analysis techniques. IEE Proceedings-Radar, Sonar and Navigation, 2004,151: 19-30.

[4] G. Davidson, H. D. Griffiths. Wavelet detection scheme for small targets in sea clutter. Electronics Letters, 2002,38: 128-1130.

[5] T. Bhattacharya, S. Haykin. Neural network-based radar detection for an ocean environment. IEEE Transactions on Aerospace and Electronic Systems, 1997,33: 408-420.

[6] S. Suvorova, B. Moran, M. Viola. Adaptive modeling of sea clutter and detection of small targets in heavy sea clutter. IEEE International Radar Conference, 2003: 614-618.

[7] B. Fridelander. A subspace framework for adaptive radar waveform design. Proc. Asilomar Conference on Signals, Systems and Computers, 2005: 1135-1195.

[8] Xie Hong-sen, Zou Kun, Zhou Peng. Statistical Analysis of Sea Clutter at Low Grazing Angle. Radar Science and Technology, 2011, 9(2):172-179 (In Chinese).

[9] K. Ward, C. Baker, and S. Watts, Maritime surveillance radar Part I: Radar scattering from the ocean surface. IEEE Proceedings F: Communications, Radar and Signal Processing, 1990, 137(4):51-62.

[10] K. J. Sangston, K. R. Gerlach. Coherent detection of radar targets in a non-Gaussian background. IEEE Transactions on Aerospace and Electronic Systems, 1994, 30(4):330-340.

[11] J. Wang, A. Dogandzic, Nehorai. Maximum likelihood estimation of compound-Gaussian clutter and target parameters. IEEE Trans. Sig. Proc, 2006, 54(10): 3884-3898.

[12] A. Dempster, N. Laird, D. Rubin. Maximum likelihood estimation from incomplete data via the EM algorithm. Journal of the Royal Statistical Society, Series B, 1977, 39(1):1-38. 